\title{
Clinical Management of Stage III Infectious Bovine Keratoconjunctivitis associated with Staphylococcus aureus in a dairy cow: A Case Report
}

\author{
1,2 *Faez Firdaus Jesse Abdullah, ${ }^{1,3}$ Lawan Adamu, ${ }^{1}$ Abdinasir Yusuf Osman \\ ${ }^{1,2}$ Abdul Wahid Haron and ${ }^{1}$ Abdul Aziz Saharee \\ ${ }^{I}$ Department of Veterinary Clinical Studies, Faculty of Veterinary Medicine, Universiti Putra Malaysia, 43400 \\ Serdang, Selangor, Malaysia, ${ }^{2}$ Research Centre for Ruminant Disease, Universiti Putra Malaysia, 43400 \\ Serdang, Selangor, Malaysia, ${ }^{3}$ Department of Veterinary Medicine, Faculty of Veterinary Medicine, University \\ of Maiduguri, PMB1069, Borno State, Nigeria
}

\begin{abstract}
A 4 year old female Friesian and Jersey cross breed dairy cattle weighing $300 \mathrm{~kg}$ was managed semi-intensively. The case was presented to the Ambulatory unit of the Large Animal Ward, University Veterinary Hospital (UVH), Universiti Putra Malaysia. There was an epiphora of the affected right eye which had increased corneal opacity with episcleral congestion. There was loss of menace reflex of the left eye which indicates loss of sight of the right eye however; there were presence of palpebral reflex on both lateral and medial aspect of the eye. Swab sample of affected right eye was taken using a sterile swab for bacterial culture, isolation and identification. The culture is a mixed growth of Staphylococcus aureus, Pantoa aglomerans, and Staphylococcus intermedius. However, there were no growth of Moraxella bovis or Mycoplasma bovis as these two organisms are the commonly isolated organism in cases of IBK. The therapy was effective and show significant improvement within 30 days of treatment. There were no epiphora observed with reduced corneal opacity and formation of scar tissue in the center of the eye which indicate healing process. Menace and palpebral reflex tests proves positive results for both of the test.
\end{abstract}

Keywords: Stage III infectious bovine keratoconjunctivitis, corneal ulcer, dairy cattle

Corresponding Author's email: jesseariasamy@gmail.com

\section{Introduction}

Infectious bovine keratoconjuctivitis or commonly known as pink eye is a contagious bacterial disease of the eye. This infection will cause the inflammation of the tissue lining the eyelid, the cornea and conjunctiva ultimately causing ulceration which may progress to produce pain and worsen causing temporary or permanent blindness.

The disease is highly contagious, it can also cause great economic impact such as reduction in growth rate of growing calves, reduction in milk production of dairy cows and also reduction in the market values of afflicted cows (Frisch, 1975).

The most common causative agent of IBK is Moraxella bovis (McConnel et al., 2007). Pathogenesis of the disease is influenced by many factors, such as season, mechanical irritation (dust, grass, weeds, etc.), host immune response, eye pigmentation, and concurrent presence of pathogenic bacteria, environment, and strain of M. bovis (Webber and Selby, 1981; Snowder, et al., 2005). Transmission of M. bovis is by direct contact, nasal and ocular discharges, and most commonly by the face fly (Brown and Adkins, 1972). Other factor that may influence the occurrence of IBK is breed differences; Hereford is more susceptible compared to other breed (Frisch, 1975; Webber and Selby, 1981). However, it is not fully known to which extent that the genetic may affect the animal as for example genetic selection and breeding will help decrease the incidence of IBK (Frisch, 1975; Webber and Selby, 1981). 


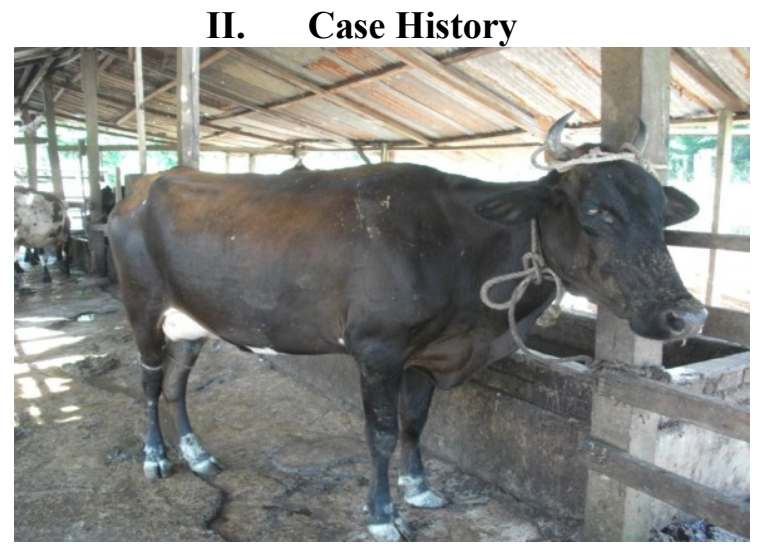

Figure 1: A case of infectious bovine keratoconjuctivitis in a cow with body score of $3 / 5$

Hitam is a 4 year old female Friesian and Jersey cross breed dairy cattle weighing $300 \mathrm{~kg}$ which was managed semi-intensively. The cattle were fed with PKC pellet in the morning and were allowed to graze in the afternoon. Milking was done twice a day in the morning and in the evening.

The owner noticed that the animal had an eye problem since $5^{\text {th }}$ of October 2011 and the he decided to treat the animal with Betamox (Amoxicillin Trihydrate) which is a broad spectrum bactericidal antibiotic and administered $20 \mathrm{ml}$ intramuscularly. Terramycin aerosol spray (2\% Oxytetracyline) was also applied.

Physical examination was carried out on the day of presentation and the temperature, pulse and respiratory rates were within the normal ranges however, there was an epiphora of the affected right eye which had increased corneal opacity with episcleral congestion (Figure 1).

Menace reflex and palpebral reflex test were done on the affected eye which results in the loss of menace reflex of the right eye which indicates loss of sight of the right eye however there was still presence of palpebral reflex on both lateral and medial aspect of the eye. It was also noticed that the farm had history of IBK previously with an average of 10 cases per year.

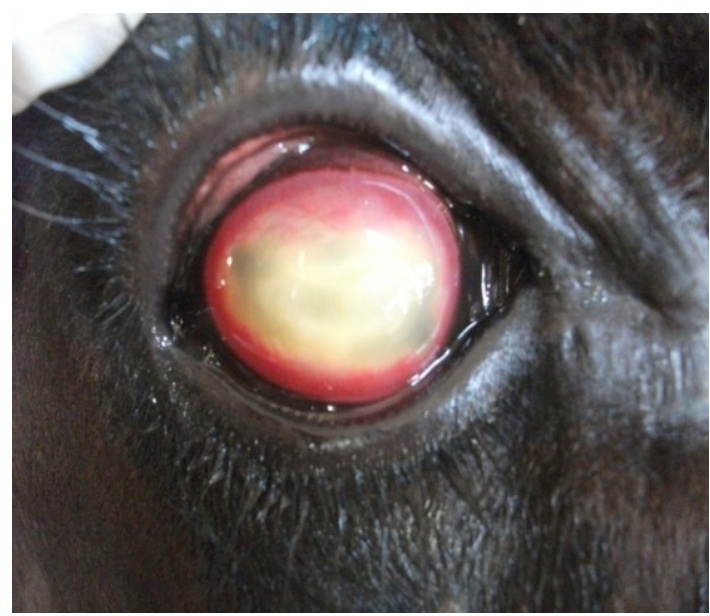

Figure 2: Infected right eye before treatment

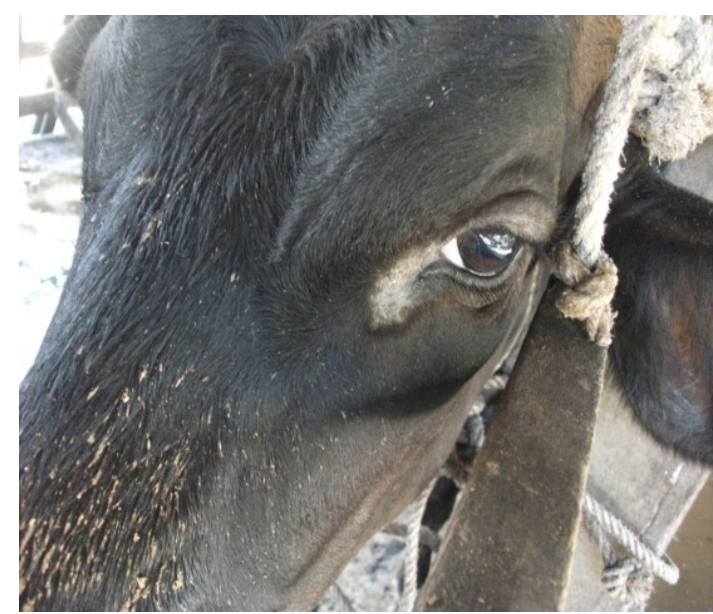

Figure 3: Normal left eye

\section{Clinical Workout}

Swab sample of the affected right eye was taken using a sterile swab and was transferred to a transport media before it was sent to the bacteriology laboratory for bacterial culture, isolation and identification.

\section{Bacterial culture, isolation and identification result}

The bacterial culture result revealed the growth of gram positive cocci (1+) and gram negative small rods (+1). The culture is a mixed growth of Staphylococcus aureus, Pantoa aglomerans, and Staphylococcus intermedius. However, there were no growth of Moraxella bovis or Mycoplasma bovis as these two organisms are the commonly isolated organism in cases of IBK. This is most probably due to the treatment done by the farmer which may have killed the organism in the eye resulting in the inability to culture and isolate the common organisms responsible for IBK in cattle.

Although Staphylococcus aureus is not a common isolated organism in cases of IBK and they are usually a normal flora of the skin but, they could be an opportunistic bacteria to have caused the lesion in the 
eye as they are pathogenic bacteria which are able to cause problem once they are provided with the point of entry into the eye. There was no literature which indicates that Staphylococcus aureus are able to cause IBK in cattle. Therefore, this could be categorized as an isolated and rare case of IBK caused by Staphylococcus aureus.

Consequently, from the bacterial culture, isolation and identification and as well as the clinical signs and history of the farm, the tentative diagnosis of infectious bovine keratoconjuctivitis was arrived.

\section{Treatment and Progression Of The Case}

\begin{tabular}{ll}
\hline Problems & \multicolumn{1}{c}{ Treatment } \\
Keratoconjuctivitis & 1. Oxytetracyline 20mg/kg 20\% IM SID \\
Corneal ulcer & 2. Aerosol spray of Oxytetracyline 2\% QID for 7 days \\
& 1. Terramycin eye ointment TID for 4 weeks \\
\hline
\end{tabular}

Antibiotic therapy started on the $12^{\text {th }}$ October 2011 with long-acting Oxytetracyline $20 \mathrm{mg} / \mathrm{kg}, 30 \mathrm{ml}$ was administered intramuscularly and an aerosol spray of Terramycin (Oxytetracyline 2\%) was applied QID (every 6 hours) for 7 days together with Terramycin eye ointment TID (every 8 hours) for a month. The topical eye ointment contains an antibiotic which helps in reducing the irritation of the eye and inhibits the progression of ulcer.

Oxytetracyline was chosen as the antibiotic of choice for the treatment as it is a broad-spectrum bacteriostatic antibiotic effective against mycoplasma, chlamydia or ricketsial organisms (Roeder et al., 2005; McConnel et al., 2007). Although there was no common agent causing IBK was culture or isolated, Oxytetracyline can be used as it is a broad-spectrum bacteriostatic antibiotic and can still be effective against Staphylococcus aureus.

The usual treatments for IBK are the topical, systemic and the sub conjunctiva treatments. In the present case report, the treatments of choice were the topical and systemic treatments. Other drugs which can be used are steroid drugs which help in reducing the inflammation of the eye such as Dexamethasone however, steroid may impair the formation of vasculature which helps in healing process; therefore it was not used in the present case report. Antibiotic such as Tylosin; a macrolide class of antibiotic which inhibits bacterial protein is ideal for use in the current case report.

The animal responded well towards the antibiotic therapy and showed significant improvement within 30 days of treatment. There were no epiphora observed with reduced corneal opacity and formation of scar tissue in the center of the eye which indicate healing process (Figure 5). Menace and palpebral reflex tests proves positive results for both of the test.

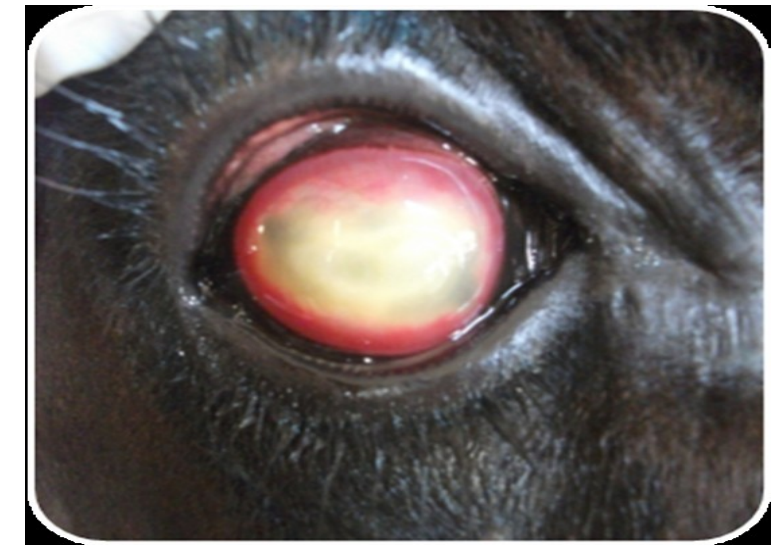

Figure 4: On the first day of presentation

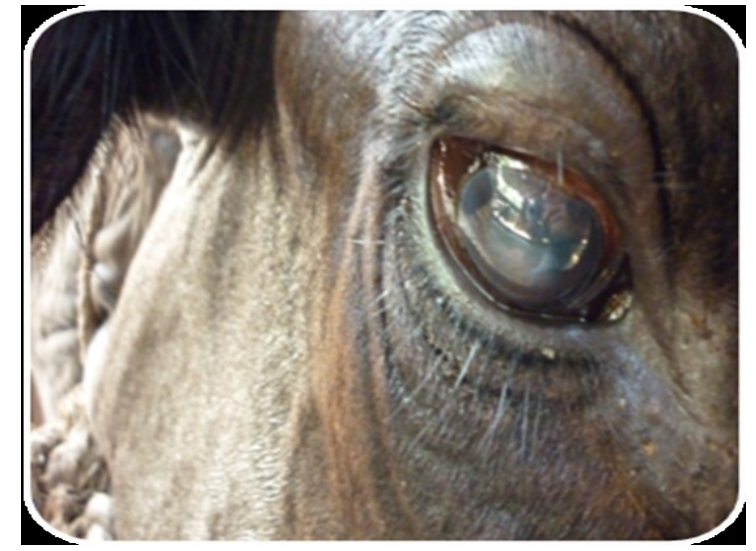

Figure 5: 30 days post treatment

\section{Discussion}

There are many predisposing factors which contributes towards the infection of IBK in cattle such as genetic and age (Webber and Selby, 1981; Ward and Nielson, 1979). Vitamin A, copper and selenium deficiencies are also another factors contributing to the infection of IBK. Vitamin A deficiency will cause the impairment of the lining of the eye (conjunctiva and sclera) and this will lead to the failure of the conjunctiva and sclera to provide protection against organism or physical agent which may cause damage to the lining of the eye. Furthermore, vitamin A helps in new cell growth, healthy skin, hair, and tissues, and vision in dim light (Snowder et al., 2005). 
Concurrent infection with viruses and Infectious bovine rhinotracheitis (IBR) and Mycoplasma bovoculi may also cause eye disease. Irritation of the eye is a major predisposing factor to pinkeye. Sunlight, dust, pollen, weed and grass seeds or awns are major eye irritants. Irritation causes primarily inflammation that allows the $M$. bovis organism to invade the tissues and multiply. Sun light allow irritation of the corneal epithelium resulting in an entry point for pathogens in to the eye.

Clinical lesions of IBK can be categorized into four stages according to their severity. Stage I is indicated when there is an excessive tearing and increased sensitivity to light which progresses to a small ulcer in the center of the cornea which appears as a small white spot (Figure 6). Stage II is indicated when the ulcer spreads across the cornea with the cornea becoming increasingly cloudy (increased opacity). Blood vessels from the outside portion of the cornea begin to grow across the cornea to help enhance healing (Figure 7).

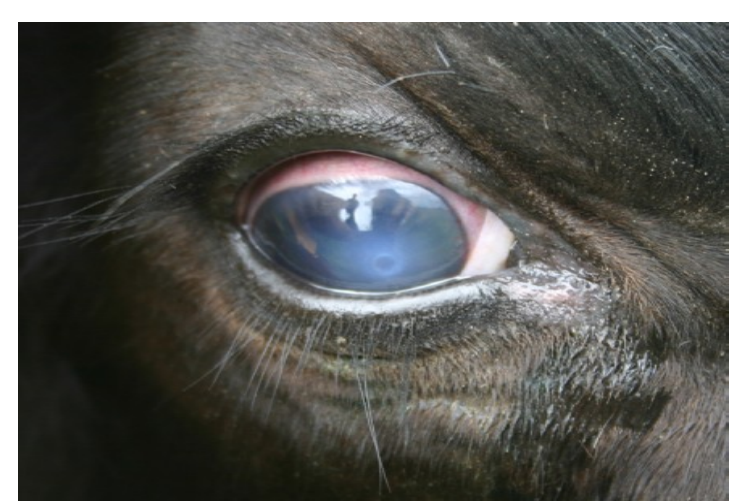

Figure 6

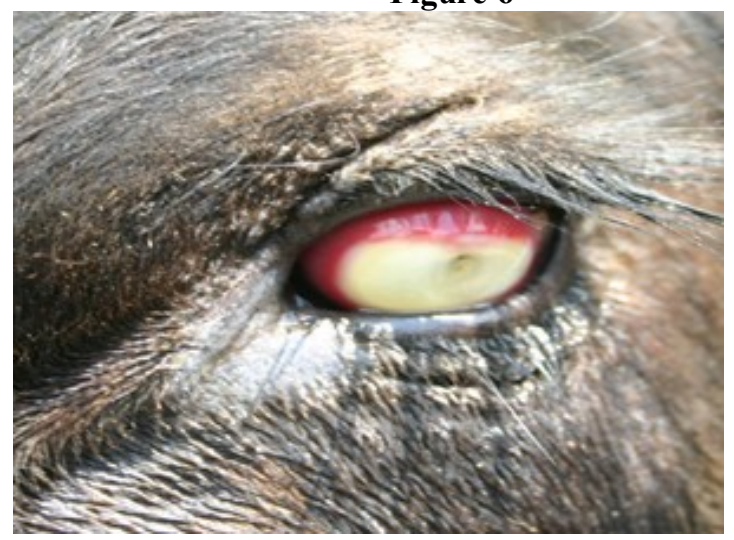

Figure 8

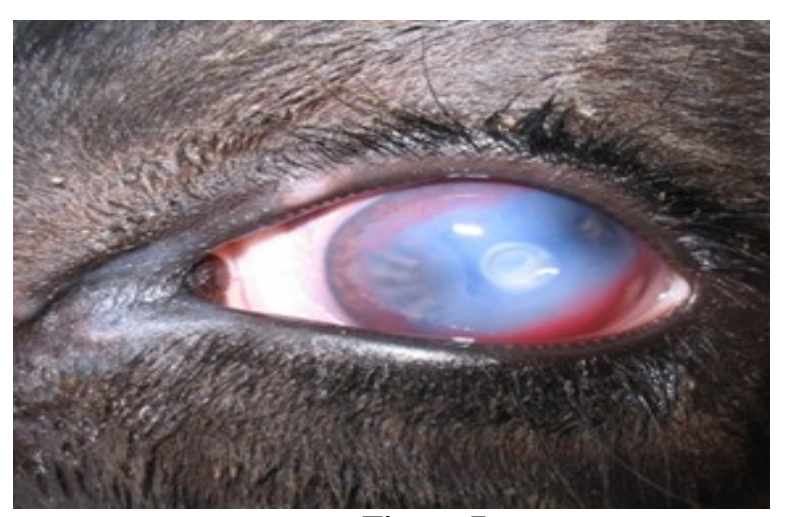

Figure 7

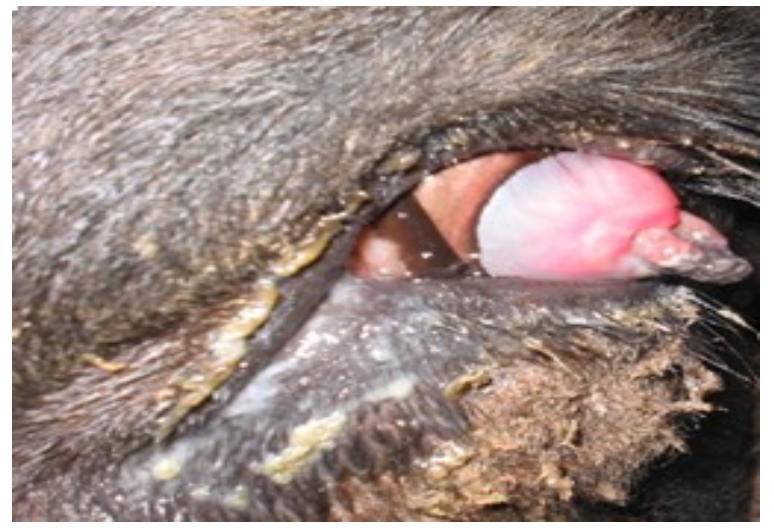

Figure 9

Stage III is indicated when the ulcer has covered almost the entire cornea and inflammation continues to spread into the inner parts of the eye. The inside of the eye is filled with fibrin, which is a pus-like substance that gives the eye a yellow appearance (Figure 8). Stage IV becomes obvious when the ulcer extends completely through the cornea, and the iris may protrude through the ulcer (Figure 9). The iris will become stuck in the cornea even after healing process has occurred (http://pubs.ext.vt.edu/400/400-750/400-750.html; Sargison et al., 1996; Whittier, et al., 2009).

\section{Control And Prevention}

Addition of vitamin A in feed may help in preventing the occurrence of IBK as vitamin A helps in the maintenance of the integrity of the surface of the eye, mucous membranes and skin are effective barriers to bacteria and viruses, reducing the risk of eye infections, respiratory problems and other infectious diseases.

Fly control is very essential in preventing IBK from occurring as the fly (face fly, stable fly, and house fly) are important vectors of the transmission of IBK pathogenic organisms. The flies usually carry the organisms and cause damage to the eye lining using their proboscis thereby creating a point of entry for the organisms to proliferate and cause IBK infection.

Vaccination may help in prevention of IBK as this will reduce the number of susceptible hosts in a herd therefore less outbreak of IBK may occur. However, vaccination program was not implemented in Malaysia but it was used in other country with epidemic of IBK infection. 
Vaccination can be done using bacterin such as pilli from organism (Moraxella bovis). Cellular vaccine comprises of vaccines developed to prevent IBK include live, killed, whole cell or subunit vaccines (McConnel and House, 2005; Funk et al., 2009).

Management of the farm also plays an important role in controlling and prevention of IBK. Hygiene of the farm, feeding management, and also isolation of infected animals or newly bought or introduce animals into the farm.

Keeping pasture cut and free of seed heads is an important part of control measure because small cuts or abrasions on the eye can allow the infectious organism to enter. Spread hay out and avoid or lower overhead feeders if possible. Prevent overcrowding at the feed bunk and provide enough shade (http://www.vetmed.ucdavis.edu/vetext/INF-BE.html).

\section{Acknowledgement}

The authors wish to acknowledge En Nazim Razali Kanini, En Mohd Jefri and Mr. Velloo of University Veterinary Hospital (UVH), and Faculty of Veterinary Medicine Universiti Putra Malaysia for their technical assistance.

\section{References}

[1] Angelos, J. A., Spinks, P.Q., Ball, L. M and George, L. W. 2007. Moraxella bovoculi sp. nov., isolated from calves with infectious bovine keratoconjunctivitis. Int J Syst Evol Microbiol. 57(4):789-95.

[2] Brown, J. F and Adkins T .R. 1972. Relationship of feeding activity of face fly (Musca autumnalis DeGeer) to production of keratoconjunctivitis in calves. Am J Vet Res; 33:2551-2555.

[3] Frisch, J. E. 1975. The relative incidence and effect of bovine infectious keratoconjunctivitis in Bos indicus and Bos taurus cattle. Anim Prod; 21:265-274

[4] Funk L, O'Connor AM, Maroney M, Engelken T, Cooper VL, Kinyon J, Plummer P. 2009. A randomized and blinded field trial to assess the efficacy of an autogenous vaccine to prevent naturally occurring infectious bovine keratoconjunctivis (IBK) in beef calves. Vaccine; $27: 4585-4590$

[5] http://pubs.ext.vt.edu/400/400-750/400-750.html

[6] http://www.vetmed.ucdavis.edu/vetext/INF-BE.html

[7] McConnel C. S and House J. K. 2005. Review article of Infectious bovine keratoconjunctivitis vaccine development. Australian Veterinary Journal. $83(8)$

[8] McConnel, C.S. Shum, L and House, J. K. 2007. Review article of Infectious bovine keratoconjunctivitis antimicrobial therapy. 85, $1 \& 2,65-69$.

[9] Roeder, B. L, Clark, F. D, Skogerboe, T. L, Kelly, E. J. 1995. Effect of early treatment with parenteral long-acting Oxytetracyline on performance of beef calves with acute eye lesions. Agri-Practice; 16:6-11.

[10] Sargison, N. D, Hutner J. E, West, D. M, Gwozdz, M. J. 1996. Observations on the efficacy of mass treatment by subconjunctival penicillin injection for the control of an outbreak of infectious bovine keratoconjunctivitis. $N Z$ Vet J. 44:142-144.

[11] Snowder, G. D, VanVleck, L. D, Cundiff, L. V, Benneth, G. L. 2005. Genetic and environmental factors associated with incidence of infectious bovine keratoconjunctivitis in preweaned beef calves. J Anim Sci; 83:507-518.

[12] Takele, G and Zerihun, A. 2000. Epidemiology of Infectious Keratoconjunctivitis in Cattle in South-east Ethiopia. J Vet Med A Physiol Pathol Clin Med.; 47(3):169-73

[13] Ward JK, Nielson MK. Pinkeye. 1979. bovine infectious keratoconjunctivitis in beef cattle. J Anim Sci; 49:361-366.

[14] Webber, J. J, Selby, L .A. 1981. Risk factors related to the prevalence of infectious bovine keratoconjunctivitis. $J$ Am Vet Med Assoc, 179:823-826.

[15] Whittier, W. D, et al. 2009. A review of Pinkeye in Beef Cattle, publication 400-750 